\title{
Implications of $\beta_{s}$ measurements
}

\section{Luca Silvestrini ${ }^{* \dagger}$}

INFN, Sez. di Roma, P.le A. Moro, 5, I-00185 Rome, Italy

E-mail: luca.silvestrinieromal.infn.it

We present an update of the analysis of $B_{s}$ mixing by the UTfit Collaboration, including the very recent tagged analyses of $B_{s} \rightarrow J / \Psi \phi$ by the CDF and DØ collaborations. We find that the phase of the $B_{s}$ mixing amplitude deviates more than $2.9 \sigma$ from the Standard Model prediction. This results points to extensions of the Standard Model with new sources of CP violation. We compare this result with the analysis of $B_{d}$ mixing and show that present data favour the presence of a new source of flavour violation.

Physics at LHC 2008

29 September - October 4, 2008

Split, Croatia

\footnotetext{
* Speaker.

${ }^{\dagger}$ Member of the UTfit Collaboration.
} 

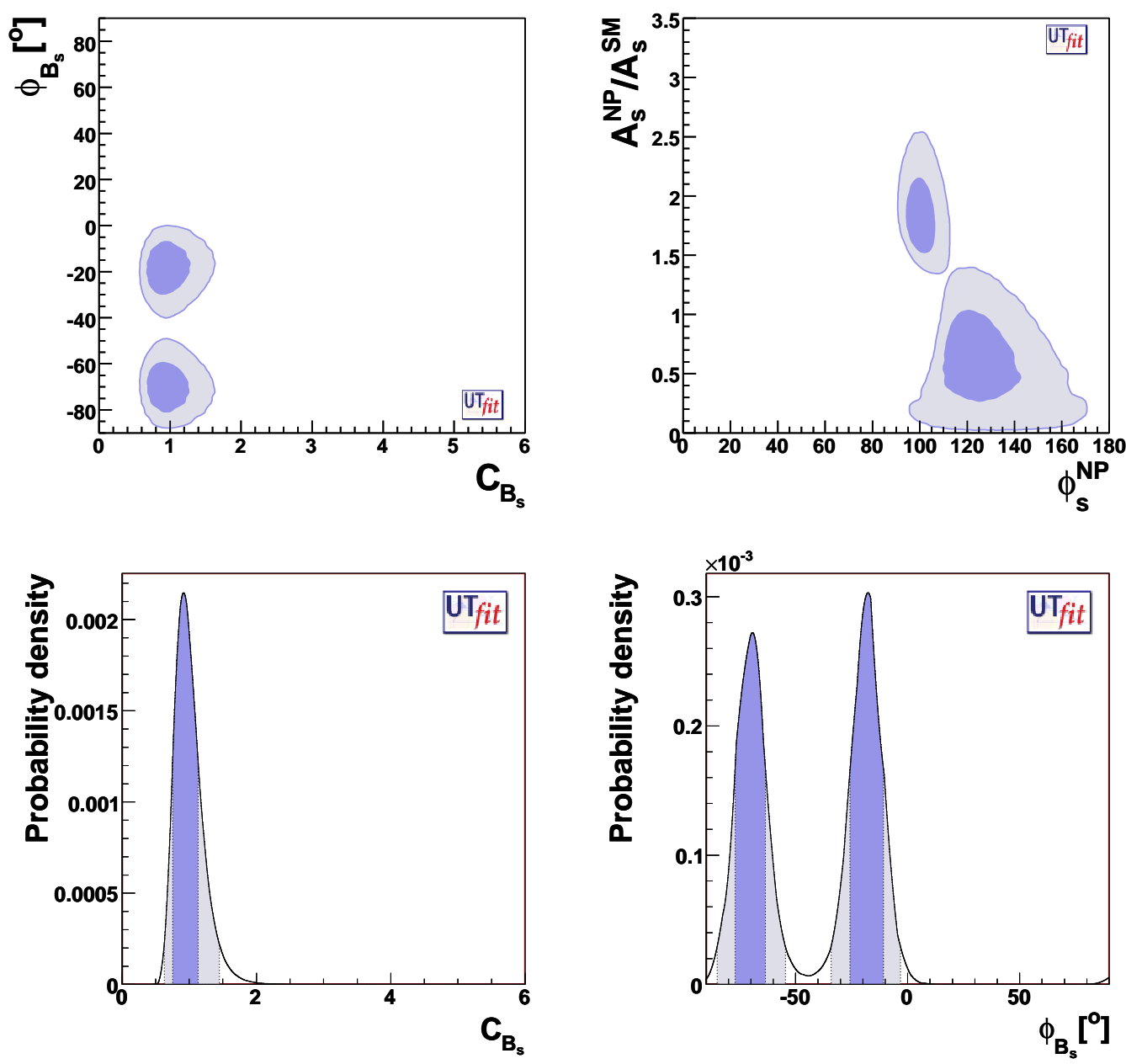

Figure 1: From left to right and from top to bottom, 68\% (dark) and 95\% (light) probability regions in the $\phi_{B_{s}}-C_{B_{s}}$ and $A_{s}^{\mathrm{NP}} / A_{s}^{\mathrm{SM}}-\phi_{s}^{\mathrm{NP}}$ planes and p.d.f for $C_{B_{s}}$ and $\phi_{B_{s}}$.

Within the Standard Model (SM), CP violation in $B_{s}$ mixing is very well predicted and small, the phase of the mixing amplitude being predicted as

$$
\sin 2 \beta_{s}=0.037 \pm 0.002 \quad[1] .
$$

The result above is also valid in extensions of the SM with Minimal Flavour Violation (MFV). Even allowing for the presence of arbitrary New Physics (NP) in all sectors, the SM contribution to the phase of the $B_{s}$ mixing amplitude is still tightly constrained:

$$
\sin 2 \beta_{s}=0.041 \pm 0.004 \quad[1] .
$$

Thus, observing a mixing phase significantly different from the value in eq. (2) would be a very clean signal of NP in $B_{s}$ mixing.

The UTfit Collaboration has recently reported evidence of a $B_{s}$ mixing phase much larger than expected in the SM, with a significance of more than $3 \sigma$ [1]. This result was obtained by 


\begin{tabular}{ccc} 
& $68 \%$ Prob. & $95 \%$ Prob. \\
\hline$\left.\phi_{B_{s}}{ }^{\circ}\right]$ & $(-19 \pm 8) \cup(-69 \pm 7)$ & {$[-36,-5] \cup[-83,-54]$} \\
$C_{B_{s}}$ & $0.94 \pm 0.19$ & {$[0.63,1.43]$} \\
\hline$A_{\mathrm{SL}}^{s} \cdot 10^{2}$ & $-0.42 \pm 0.23$ & {$[-0.90,0.01]$} \\
$A_{\mathrm{SL}}^{\mu \mu} \cdot 10^{3}$ & $-2.5 \pm 1.1$ & {$[-5.1,-0.5]$} \\
$\Delta \Gamma_{s} / \Gamma_{s}$ & $(0.13 \pm 0.06) \cup(-0.12 \pm 0.05)$ & {$[0.02,0.23] \cup[-0.22,-0.02]$} \\
\hline
\end{tabular}

Table 1: Fit results for NP parameters, semileptonic asymmetries and width differences. Whenever present, we list the two solutions due to the ambiguity of the measurements. The first line corresponds to the one closer to the SM.

combining all available experimental information with the method used by the UTfit Collaboration for UT analyses and described in Ref. [2]. We present here an update of this analysis, including the very recent data presented at the summer conferences. We refer the reader to Ref. [1] for the details of the analysis.

We perform a model-independent study of NP contributions to $B_{s}$ mixing using the following parametrization [3]:

$$
C_{B_{s}} e^{2 i \phi_{B_{s}}}=\frac{A_{s}^{\mathrm{SM}} e^{-2 i \beta_{s}}+A_{s}^{\mathrm{NP}} e^{2 i\left(\phi_{s}^{\mathrm{NP}}-\beta_{s}\right)}}{A_{s}^{\mathrm{SM}} e^{-2 i \beta_{s}}}=\frac{\left\langle B_{s}\left|H_{\mathrm{eff}}^{\mathrm{full}}\right| \bar{B}_{s}\right\rangle}{\left\langle B_{s}\left|H_{\mathrm{eff}}^{\mathrm{SM}}\right| \bar{B}_{s}\right\rangle},
$$

where $H_{\mathrm{eff}}^{\text {full }}$ is the effective Hamiltonian generated by both SM and NP, while $H_{\mathrm{eff}}^{\mathrm{SM}}$ only contains SM contributions.

We use the following experimental input: the CDF measurement of $\Delta m_{s}$ [4], the semileptonic asymmetry in $B_{s}$ decays $A_{\mathrm{SL}}^{s}$ [5], the dimuon charge asymmetry $A_{\mathrm{SL}}^{\mu \mu}$ from DØ [6] and CDF [7], the measurement of the $B_{s}$ lifetime from flavour-specific final states [8], the two-dimensional likelihood ratio for $\Delta \Gamma_{s}$ and $\phi_{s}=2\left(\beta_{s}-\phi_{B_{s}}\right)$ from the time-dependent tagged angular analysis of $B_{s} \rightarrow J / \psi \phi$ decays by CDF [9] and DO [10]. The values for input parameters can be found in Ref. [1], except for $A_{\mathrm{SL}}^{s}$ for which we use the new value from $\mathrm{D} \emptyset A_{\mathrm{SL}}^{s}=-0.0020 \pm 0.0119$ and for the tagged analysis by $\mathrm{D} \emptyset$, for which we use the new analysis performed with no assumption on the strong phase.

The results of our analysis are summarized in Table 1. The phase $\phi_{B_{s}}$ deviates from zero at $99.6 \%$ probability, equivalent to $2.9 \sigma$. In Fig. 1 we present the two-dimensional $68 \%$ and 95\% probability regions for the NP parameters $C_{B_{s}}$ and $\phi_{B_{s}}$ and the one-dimensional distributions for NP parameters. Notice that the ambiguity of the tagged analysis of $B_{s} \rightarrow J / \Psi \phi$ is slightly broken by the presence of the CKM-subleading terms in the expression of $\Gamma_{12} / M_{12}$ (see for example eq. (5) of ref. [11]). The solution around $\phi_{B_{s}} \sim-20^{\circ}$ corresponds to $\phi_{s}^{\mathrm{NP}} \sim-50^{\circ}$ and $A_{s}^{\mathrm{NP}} / A_{s}^{\mathrm{SM}} \sim 75 \%$ (see Fig. 1 . The second solution is much more distant from the SM and it requires a dominant NP contribution $\left(A_{s}^{\mathrm{NP}} / A_{s}^{\mathrm{SM}} \sim 190 \%\right)$. In this case the NP phase is thus very well determined. The strong phase ambiguity affects the sign of $\cos \phi_{s}$ and thus $A_{s}^{\mathrm{NP}} / A_{s}^{\mathrm{SM}} \cos \phi_{s}^{\mathrm{NP}}$, while $A_{s}^{\mathrm{NP}} / A_{s}^{\mathrm{SM}} \sin \phi_{s}^{\mathrm{NP}} \sim-0.74$ in any case.

To illustrate the impact of the experimental constraints, we show in Fig. 2 the p.d.f. for $\phi_{B_{s}}$ obtained with various subsets of experimental constraints. Including only the CDF tagged analysis, we obtain $\phi_{B_{s}}=(-25 \pm 11)^{\circ} \cup(-63 \pm 11)^{\circ}\left([-83,-5]^{\circ}\right.$ at $95 \%$ probability $)$. The SM value $\phi_{B_{s}}=0$ 

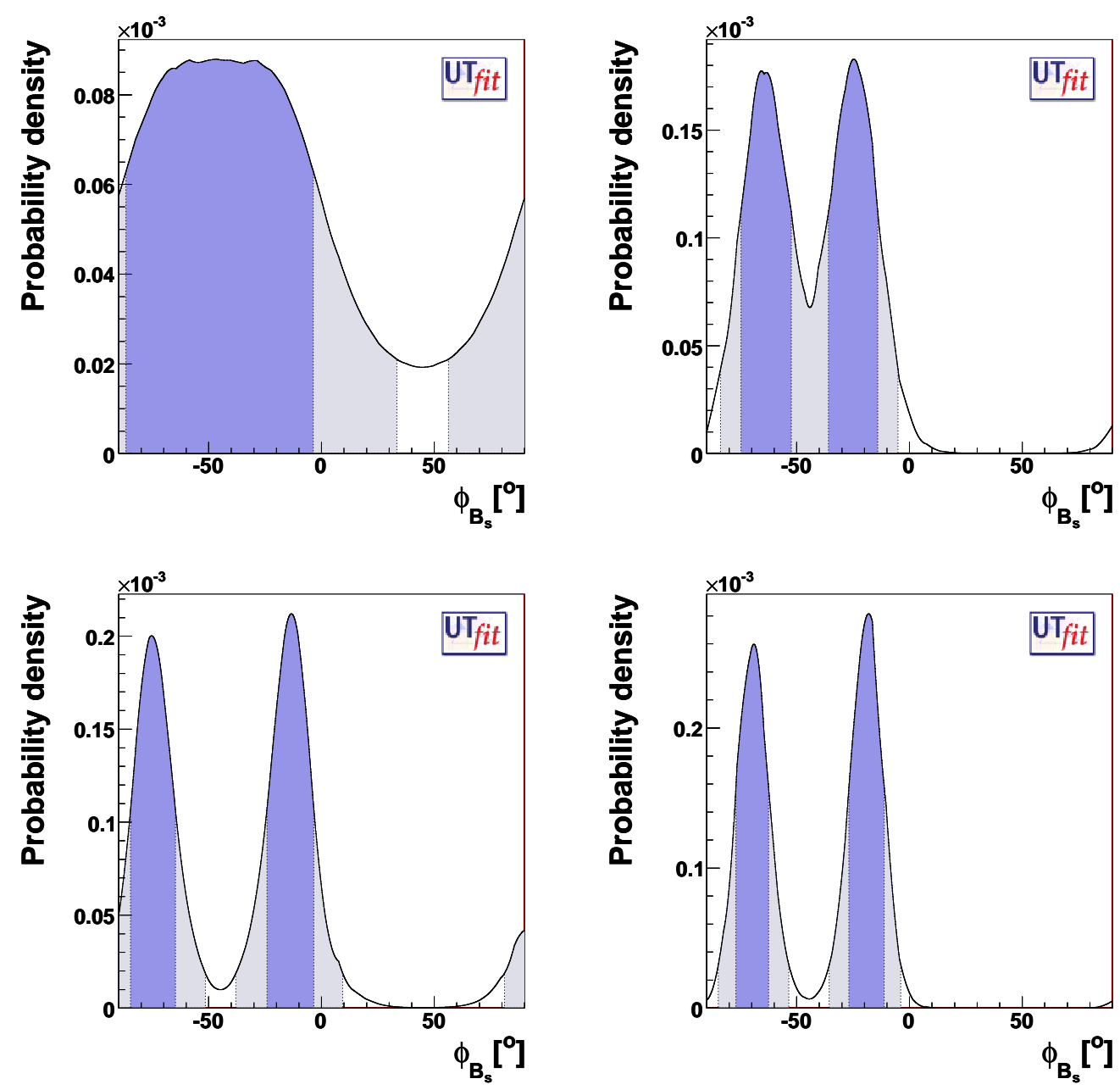

Figure 2: From left to right and from top to bottom, p.d.f. for $\phi_{B_{s}}$ without the tagged analysis of $B_{s} \rightarrow J / \Psi \phi$, including only the CDF analysis, including only the $\mathrm{D} \emptyset$ analysis, including only the tagged analysis of $B_{s} \rightarrow J / \Psi \phi$ from both experiments. We show $68 \%$ (dark) and 95\% (light) probability regions.

is only present in the $98 \%$ probability range $(2.4 \sigma)$. Using only the DØ tagged analysis, we get $\phi_{B_{s}}=(-14 \pm 10)^{\circ} \cup(-75 \pm 10)^{\circ}\left([-99,-52]^{\circ} \cup[-38,10]^{\circ}\right.$ at $95 \%$ probability $)$, and the SM is included in the $81 \%$ probability range $(1.3 \sigma)$. Using both analyses, we obtain $\phi_{B_{s}}=(-19 \pm 8)^{\circ} \mathrm{U}$ $(-70 \pm 7)^{\circ}\left([-84,-54]^{\circ} \cup[-36,-4]^{\circ}\right.$ at $95 \%$ probability $)$, and the SM is included in the $99 \%$ probability range $(2.6 \sigma)$. Semileptonic asymmetries alone give $\phi_{B_{s}}=(-45 \pm 42)^{\circ}\left([-124,33]^{\circ}\right.$ at $95 \%$ probability). We stress that the different constraints in Fig. 2 are all consistent among themselves and with the combined result. For completeness, in Table 1 we also quote the fit results for $A_{\mathrm{SL}}^{s}, A_{\mathrm{SL}}^{\mu \mu}$ and for $\Delta \Gamma_{s} / \Gamma_{s}$.

It is remarkable that to explain the result obtained for $\phi_{s}$, new sources of CP violation beyond the CKM phase are required, strongly disfavouring the MFV hypothesis. These new phases will in general produce correlated effects in $\Delta B=2$ processes and in $b \rightarrow s$ decays. These correlations cannot be studied in a model-independent way, but it will be interesting to analyse them in the 
MSSM. Before doing so, we comment on the hierarchy in NP contributions to $\Delta F=2$ transitions required by present data. From ref. [12] we learn that the NP contribution to $B_{d}$ mixing cannot exceed $40 \%$ of the SM one if NP carries a phase around $130^{\circ}$ as required by $B_{s}$ mixing. This is marginally compatible with the $B_{s} \mathrm{NP}$ amplitude around $70 \%$ of the SM as obtained above. Thus, if NP is present in $B_{s}$ mixing, we must have $A_{d}^{\mathrm{NP}} / A_{s}^{\mathrm{NP}} \times A_{s}^{\mathrm{SM}} / A_{d}^{\mathrm{SM}} \sim \lambda_{c}$, where $\lambda_{c}$ is the Cabibbo angle, rather than $\mathscr{O}(1)$ as expected in models of Next-to-Minimal Flavour violation, in which NP contributions enjoy the same Cabibbo suppression as SM ones. We conclude that the NP causing the observed deviation in $\Phi_{B_{s}}$ must have a nontrivial flavour structure to suppress NP contributions to $B_{d}$ and $K$ mixing more than what expected from a SM-like Cabibbo structure.

It is a pleasure to thank all the UTfit Collaboration members for a most enjoyable collaboration. We acknowledge partial support from RTN European contracts MRTN-CT-2004-503369 "The Quest for Unification”, MRTN-CT-2006-035482 "FLAVIAnet” and MRTN-CT-2006-035505 "Heptools". L.S. is associated to the Dipartimento di Fisica, Università di Roma "La Sapienza".

\section{References}

[1] M. Bona et al. [UTfit Collaboration], arXiv:0803.0659 [hep-ph].

[2] M. Ciuchini et al., JHEP 0107, 013 (2001) [arXiv:hep-ph/0012308].

[3] J. M. Soares and L. Wolfenstein, Phys. Rev. D 47, 1021 (1993); T. Goto, N. Kitazawa, Y. Okada and M. Tanaka, Phys. Rev. D 53, 6662 (1996) [arXiv:hep-ph/9506311]; N. G. Deshpande, B. Dutta and S. Oh, Phys. Rev. Lett. 77, 4499 (1996) [arXiv:hep-ph/9608231]; J. P. Silva and L. Wolfenstein, Phys. Rev. D 55, 5331 (1997) [arXiv:hep-ph/9610208]; A. G. Cohen et al., Phys. Rev. Lett. 78, 2300 (1997) [arXiv:hep-ph/9610252]; Y. Grossman, Y. Nir and M. P. Worah, Phys. Lett. B 407, 307 (1997) [arXiv:hep-ph/9704287].

[4] A. Abulencia et al. [CDF Collaboration], Phys. Rev. Lett. 97, 242003 (2006) [arXiv:hep-ex/0609040].

[5] V. M. Abazov et al. [DØ Collaboration], Phys. Rev. Lett. 98, 151801 (2007) [arXiv:hep-ex/0701007].

[6] V. M. Abazov et al. [DØ Collaboration], Phys. Rev. D 74, 092001 (2006) [arXiv:hep-ex/0609014].

[7] CDF Collaboration, CDF note 9015.

[8] D. Buskulic et al. [ALEPH Collaboration], Phys. Lett. B 377, 205 (1996); F. Abe et al. [CDF Collaboration], Phys. Rev. D 59, 032004 (1999) [arXiv:hep-ex/9808003]; P. Abreu et al. [DELPHI Collaboration], Eur. Phys. J. C 16, 555 (2000) [arXiv:hep-ex/0107077]; K. Ackerstaff et al. [OPAL Collaboration], Phys. Lett. B 426, 161 (1998) [arXiv:hep-ex/9802002]; V. M. Abazov et al. [DØ Collaboration], Phys. Rev. Lett. 97, 241801 (2006) [arXiv:hep-ex/0604046]; CDF Collaboration, CDF note 7386; CDF Collaboration, CDF note 7757; E. Barberio et al. [HFAG], arXiv:hep-ex/0603003; CDF Collaboration, CDF note 9203.

[9] T. Aaltonen et al. [CDF Collaboration], arXiv:0712.2397 [hep-ex].

[10] V. M. Abazov et al. [DØ Collaboration], arXiv:0802.2255 [hep-ex].

[11] M. Bona et al. [UTfit Collaboration], Phys. Rev. Lett. 97, 151803 (2006) [arXiv:hep-ph/0605213].

[12] M. Bona et al. [UTfit Collaboration], JHEP 0803 (2008) 049 [arXiv:0707.0636 [hep-ph]]. 